\title{
Optical frequency microcomb distillation for super channel data transmission
}

\author{
Chawaphon Prayoonyong, Andreas Boes, Xingyuan Xu, Mengxi Tan, Sai T. Chu, Brent E. Little, \\ Roberto Morandotti, Fellow OSA, Fellow, IEEE, Arnan Mitchell, David J. Moss, Fellow OSA, Fellow, \\ IEEE, and Bill Corcoran
}

\begin{abstract}
Microcombs provide a potential compact and efficient light source for multi-Terabit-per-second optical superchannels. However, as the bandwidth of these multiwavelength light sources is increased, this can result in low perline power. Optical amplifiers can be used to overcome power limitations, but the accompanying spontaneous optical noise can degrade performance in optical systems. To overcome this issue, we propose wideband noise reduction for comb lines using a high$Q$ microring resonator, whose resonances align with comb lines. When applying the proposed distillation to a superchannel system with 18 Gbaud, 64-QAM sub-channels in a > $10 \mathrm{~Tb} / \mathrm{s}$ optical superchannel, we find that noise-corrupted comb lines can reduce the optical signal-to-noise ratio required for the comb by $\sim 9 \mathrm{~dB}$ when used as optical carriers at the transmitter side, and by $\sim 12$ $\mathrm{dB}$ when used as a local oscillator at the receiver side.
\end{abstract}

Index Terms-Coherent optical system, Microresonator, Narrowband filtering, Optical frequency combs, OSNR penalty

\section{INTRODUCTION}

$\mathrm{O}$ PTICAL frequency combs are unique light sources consisting of precisely equidistant frequency lines and have enabled many applications ranging from spectroscopy [1]-[3] to neural networks [4], [5], and coherent optical communications systems [6]. Although frequency comb sources are commercially available, these benchtop solutions may not be suitable for some applications, due to limitations in size, weight and power. These limitations can be potentially overcome with miniaturized 'microcombs' (reviewed in [7][9]), which have been proven in research as appropriate for a range of applications, including microwave photonics [11], [12] or optical communications [13]-[15].

In optical communication systems, the individual optical

This paragraph of the first footnote will contain the date on which you submitted your paper for review. This paragraph of the first footnote will contain the date on which you submitted your paper for review. This work was supported in part by the Australian Research Council (ARC) under Grant DP190102773.

C. Prayoonyong and B. Corcoran are with Photonic Communications Laboratory, Dept. Electrical and Computer Systems Engineering, Monash University, Clayton, VIC 3800, Australia (e-mail: chawaphon.prayoonyong @ monash.edu; bill.corcoran@monash.edu).

A. Boes and A. Mitchell are with School of Engineering, RMIT University, Melbourne, VIC 3001, Australia (e-mail: andreas.boes@rmit.edu.au; arnan.mitchell@rmit.edu.au).

M. Tan and D.J. Moss are with the Optical Sciences Centre, Swinburne University, Hawthorne, VIC 3122, Australia (e-mail: mengxitan@ swin.edu.au; dmoss@swin.edu.au). frequency comb lines can be deployed as carrier at the transmitter, or as a local oscillator (LO) at the receiver, to support superchannel transmission. High capacity optical superchannels have potential to serve future data capacity requirements in existing fiber links, where high spectral efficiency is needed to increase capacity of systems with limited optical bandwidth over a single optical mode. In these superchannel systems, optical frequency combs may provide an attractive alternatively to using a large number of conventional external cavity lasers (ECLs) to support many individual subbands. Furthermore, optical frequency combs may offer advantages in terms of simplifying signal processing while reducing power consumption and costs. Most optical frequency combs with wide bandwidth can be generated from an input laser, through nonlinear frequency conversion (e.g. [10], [13], [16], [17]) or electro-optic effect (e.g. [18]-[20]). Both methods distribute the power of the initial laser line to sidebands, hence, more sideband lines or a lower seeding power can lead to lower per-line power [21]. In addition, the generated frequency combs often have a nonuniform power distribution for the comb spectrum, which can be undesirable and it might be preferable to flatten the comb profile using an optical filter whose insertion loss could also degrade per-line power [6], [13]-[15]. To solve this problem, one can amplify comb lines using optical amplifiers such as Erbium-doped fiber amplifier (EDFA), however optical noise stemming from amplified spontaneous emission (ASE) can contaminate the amplified lines. In optical communications systems, this broadband noise could degrade the performance of comb lines when used as either carriers or LOs, especially, in high modulation formats such as 64QAM where high signal-to-noise ratios are required.

There are approaches to purify comb lines and reduce optical noise of the amplified comb techniques such as optical injection

$\mathrm{X}$. Xu was with Centre for Micro-Photonics, Swinburne University, Hawthorne, VIC 3122, Australia. He is now with Photonic Communications Laboratory, Dept. Electrical and Computer Systems Engineering, Monash University, Clayton, VIC 3800, Australia (e-mail: mike.xu@ monash.edu)

S. T. Chu is with Department of Physics, City University of Hong Kong, Tat Chee Ave, Hong Kong, China (e-mail: saitchu@cityu.edu.hk)

B. E. Little is with Xi'an Institute of Optics and Precision Mechanics of the Chinese Academy of Sciences, Xi'an, China (email: b.e.little@ciompxian.edu.cn)

R. Morandotti is with INRS - EMT, Varennes, Quebec J3X 1S2, Canada \& Adjunct with the Institute of Fundamental and Frontier Sciences, University of Electronic Science and Technology of China, Chengdu 610054, China (e-mail : morandotti@emt.inrs.ca) 
locking [22] or stimulated Brillouin scattering (SBS) [23], [24]. However, these approaches experience difficulties due to size, power consumption, and/or limited range in frequency.

Here, we adapt an approach employed in spectroscopy and microwave photonics [25], to filter a large number of amplified comb lines in parallel based on a high-quality-factor microring resonator $\left(\mathrm{Q} \sim 10^{6}\right)$, before using these comb lines in optical communications systems. Our results show that the performance of the distilled comb lines is significantly better than the noisy ones as they provide a reduction in the required optical signal-to-noise ratio (OSNR) by $\sim 9 \mathrm{~dB}$ and $\sim 12 \mathrm{~dB}$ at a $5 \%$ reduction of information rate, when exploited as carriers and local oscillators, respectively, with a 64QAM format. This work extends upon preliminary results presented at OFC 2020 [26]. These results suggest that passive filtering with a high $Q$ resonator can significantly improve the performance of systems using low-power optical frequency combs as a light source, providing a path toward improving the performance of power constrained combs, such as those generated efficiently by a micro-photonic resonator.

\section{PROOF OF CONCEPT FOR COMB DISTILLATION}

Amplification of low power frequency combs using an EDFA can contaminate the comb lines with optical noise, degrading performance in optical systems. In optical communications systems, such noise can degrade the overall system performance especially when advanced modulation formats are used. It has been shown that microring resonators provide an interesting and compact filtering solution for optical frequency combs, with the drop port of a double-bus ring providing a periodic inverse-notch profile [27]. Here we propose the use of a high-quality-factor Hydex microring resonator $\left(\mathrm{Q} \sim 10^{6}\right)$ [28], [29] with a bandwidth of $<200 \mathrm{MHz}$ to act as a narrowband filter for each comb line. In order to effectively distill comb lines simultaneously. It is crucial to match line spacing with the FSR of the microring resonator, so that the comb lines are allowed to pass, while broadband noise
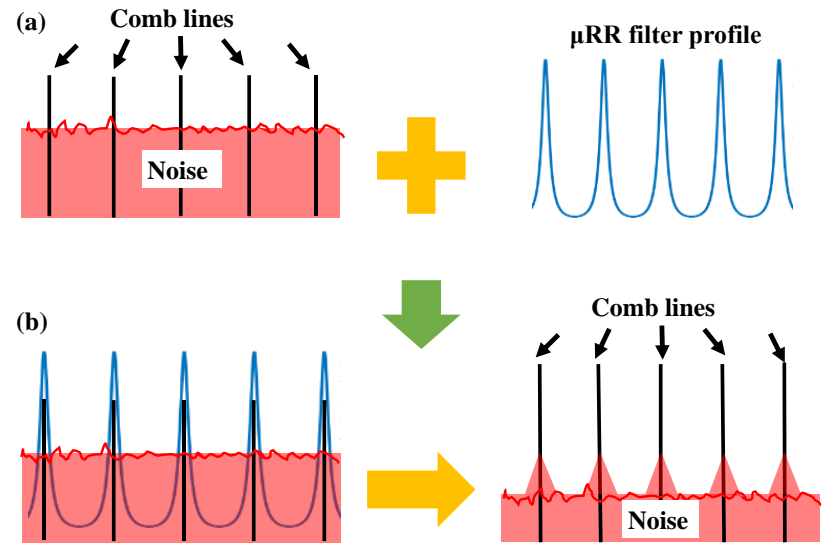

Fig. 1 (a) Comb lines with high noise floor (black and red, left) and the drop port filter profile (blue, right), (b) Filtering by microring resonator. The filter profile superimposed on the noisy comb (left) produces a noise reduced "distilled" output (black and red, right). is rejected. Fig. 1 (a)-(b) illustrates filtering by microring resonator, where comb lines are shown in black with a high noise floor (red), along with the resonator response shown with a blue curve, and the output at the drop port with a small portion of noise inside the resonance bandwidth around the comb lines remaining after distillation.

To demonstrate distillation, we generate an electro-optic frequency comb using a set of phase modulators cascaded with an intensity modulator [19], before transmitting through the microring resonator. Here, it is important to match the central wavelength and line spacing of the comb with a resonance peak and FSR of the ring, respectively, in order to minimize loss for the comb lines while attenuating noise. The details for this procedure will be discussed in the next section.

Once the comb is adjusted to satisfy conditions for resonance peaks and FSR, we then compare the spectrum of the comb before and after distillation. Fig. 2 shows two optical spectra measured with $12.5 \mathrm{GHz}(0.1 \mathrm{~nm})$ resolution, an amplified (or noisy) comb in blue, and a distilled comb in magenta, with a bandwidth of $\sim 1.3 \mathrm{THz}$. It can be seen that the noise floor for the amplified comb is $\sim-20 \mathrm{dBm}$ whereas its per-line power is on average $\sim-10 \mathrm{dBm}$, resulting in OSNR of $\sim 10 \mathrm{~dB}$. The slight tilt of the noise floor is caused by gain tilt due to the fact that EDFAs can have a frequency-dependent response if not optimally run, and that the input light is at lower power than specified for the EDFA [30].

After distillation via the ring, the out-of-band noise, as measured with a $12.5 \mathrm{GHz}$ resolution, is significantly reduced compared to the comb lines, highlighting the reduction in passed noise bandwidth by the rings. Comparing comb amplitude to noise amplitude at either end of the spectrum, this is improved by around $20 \mathrm{~dB}$, which is about what could be expected from the difference between the ring resonance bandwidth (around $150 \mathrm{MHz}$ ) to the optical spectrum measurement bandwidth $(12.5 \mathrm{GHz})$.

This indicates that broadband noise can be significantly reduced by micro-ring filtering. In the context of an optical communications system, this may be used to improve the

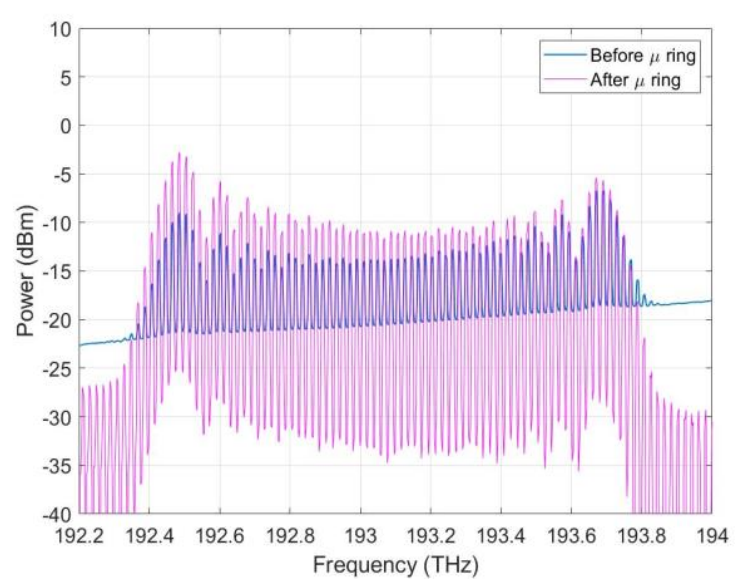

Fig. 2 Spectra for amplified (blue) comb before being distillation and distilled (magenta) EO comb. 


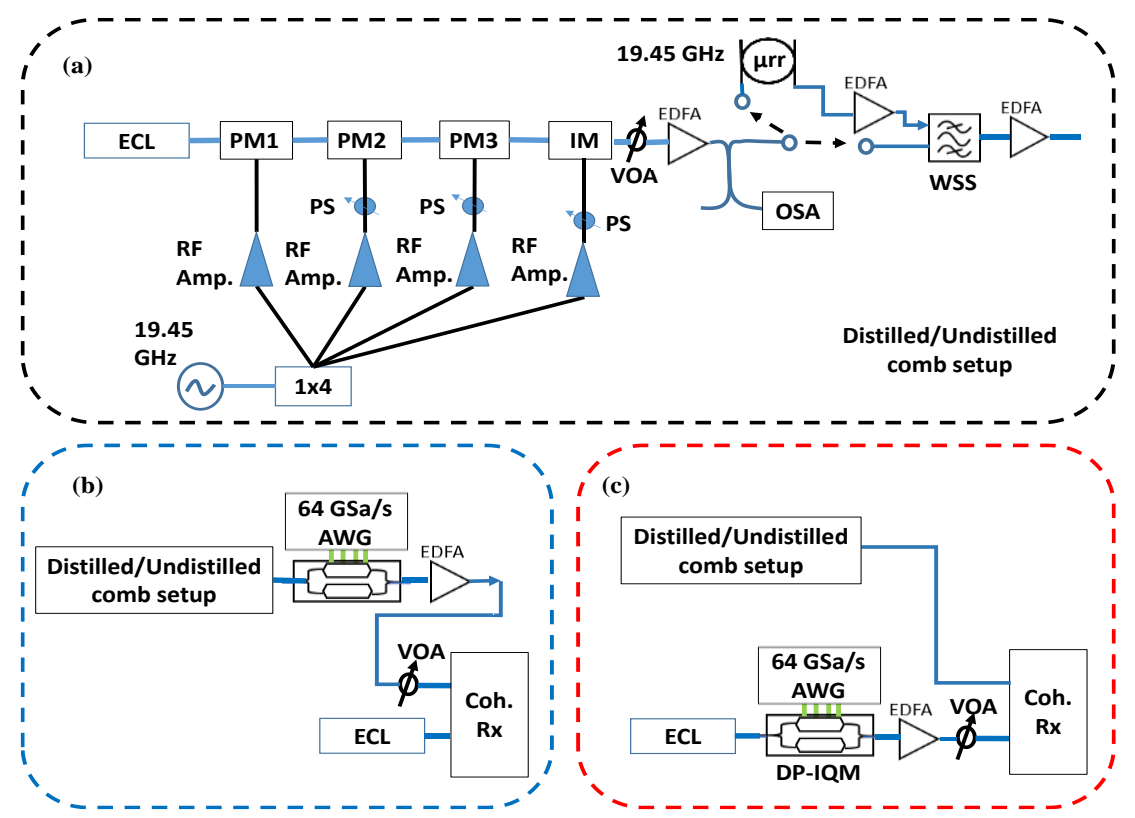

Fig. 3 (a) distilled/undistilled comb source, and demonstration when using the comb source as (b) carriers, (c) LOs; here ECL: External cavity laser, PM: Phase modulator, IM: Intensity modulator, PS: Phase shifter, OSA: optical spectrum analyzer, WSS: Wavelength selective switch, VOA: Variable optical attenuator, $\mu$ rr: Microring resonator, AWG: Arbitrary waveform generator, DP-IQM: dual-polarization I/Q modulator, Coh. Rx.: Coherent receiver

quality of optical carriers derived from low power comb sources at the transmitter and receiver. In the next sections, we will report the experimental setup when using the noisy and purified combs in 64QAM transmission and associated results.

\section{SySTEM CONFIGURATIONS}

To investigate the benefits of comb distillation in both transmit and receive configurations, we set up the experiment as shown in Fig. 3 (a)-(c). Here, a frequency comb is generated by cascaded three phase modulators and one intensity modulator (Fig. 3 (a)), to achieve the comb bandwidth as shown in Fig. 2. In this setup, the three phase modulators are employed to broaden the optical spectrum while another intensity modulator is used to flatten the spectrum as demonstrated in [19]. These modulators are driven with APW/265-3335 amplifiers by CTT Inc. In order to transmit the frequency comb through the microring resonator with a minimum loss, we need to match the comb lines to the resonant transmission peaks of the ring resonator. In principle, this could be achieved on a single photonic chip by matching resonator FSRs. As we have a separate comb and resonator in this demonstration, this matching must be ensured manually.

Initially, we sweep the frequency of a $\mathrm{CW}$ signal launched to the resonator in order to find a resonance peak, here located at 193.089 THz. We then set the CW laser to this frequency as the central frequency of the comb. After finding this initial resonance, to align the comb lines to the resonances, we search for the FSR of the resonator by launching optical noise into the resonator before measuring the response with a $150 \mathrm{MHz}$ resolution optical spectrum analyser (OSA). We find that FSR extracted from the OSA is $\sim 19.5 \mathrm{GHz}$ and, by fine tuning the comb spacing, we refine this to an FSR of $\sim 19.45 \mathrm{GHz}$. Note that the resonator is polarization and temperature dependent, thus a polarization controller, and thermistor \& thermo-electric cooler (monitored by a controller, TED200C), are deployed to align comb polarization with the resonator and stabilize the resonator frequency response.

After matching the comb to the resonator, we noise load the comb by attenuating comb, from -10 to $-30 \mathrm{dBm}$ total power, before amplification with an EDFA to bring the comb to 21 $\mathrm{dBm}$ total comb power. To measure the OSNR of the comb after this stage, $50 \%$ of the comb power is coupled to an OSA with $0.1 \mathrm{~nm}$ resolutuon. In the meantime, the remaining signal is transmitted to the microring resonator, whose drop port is connected to the second amplifier and then a wavelength selective switch (WSS), so that the comb line of interest can be used for the optical communications systems. We note that the system is configured to also allow the noise-loaded comb to be bypassed to enable performance comparison. From here, the comb line is either deployed as a carrier line at the transmitter with the ECL laser used as LO line or vice versa (see Fig 3 (b) and (c)). Here, the carrier power before the IQ modulator is set to $11 \mathrm{dBm}$, and is changed to $14 \mathrm{dBm}$ when used as an LO for coherent reception.

For the optical communications systems, a $23-\mathrm{GHz}$ bandwidth dual-polarization (DP) IQ modulator is driven by a 64-GSa/s, 25-GHz bandwidth arbitrary waveform generator with a $2.5 \%$ RRC, 64-QAM signal at $18 \mathrm{GBd}$ to emulate superchannel transmission from this $19.45 \mathrm{GHz}$ line spacing comb. Once the optical signal reaches the receiver, it is mixed with the corresponding LO line at the $25-\mathrm{GHz}$ bandwidth coherent receiver. The signal is then sampled by an $80 \mathrm{GSa} / \mathrm{s}$ oscilloscope with $33 \mathrm{GHz}$ bandwidth. Subsequently, the digitized signal is processed with DSP algorithms consisting of IQ imbalance compensation, frequency offset compensation, matched filtering, before training-based frame synchronization, equalization, and phase compensation. Following these DSP steps, signal quality, $\mathrm{Q}^{2}$, which is related to error vector magnitude $(E V M)$ as $\mathrm{Q}^{2}=1 / \mathrm{EVM}^{2}$, and bit error ratio (BER) are calculated. In addition, generalized mutual information (GMI) is also calculated with the calcGMI.m script to convey 
information

rates

https://www.fehenberger.de/\#sourcecode).

\section{DEMOnStration of COMB Distillation FOR CARriers}

In this section, we investigate and compare performance of comb lines when exploited as carriers for superchannel transmission before and after distillation with the microring resonator.

\section{A. Without distillation}

In order to compare noise reduction for carrier lines from comb distillation, we first evaluate the performance of the comb lines without distillation. Here, the comb lines tested in the experiment are marked by the red arrow as shown in Fig. 4

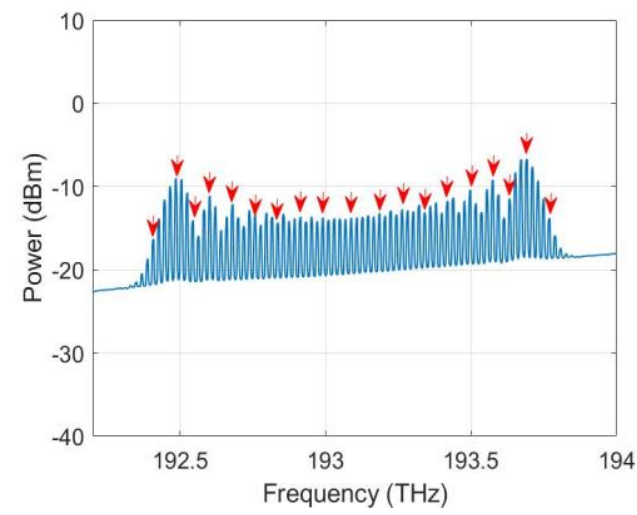

Fig.4 Electro-optic frequency comb after attenuation and amplification with the red arrows representing the lines used in the experiment.

Fig. 5 shows $\mathrm{Q}^{2}$, BER and GMI plotted against comb line OSNR for the comb lines indicated in Fig. 4, without employing comb distillation through filtering with the micro-ring resonator. In this case, the comb lines are used as optical

(a)

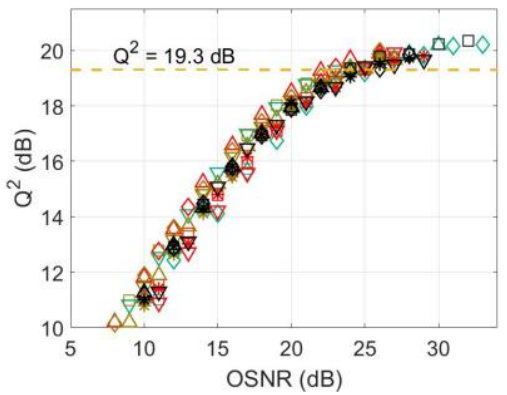

(c)

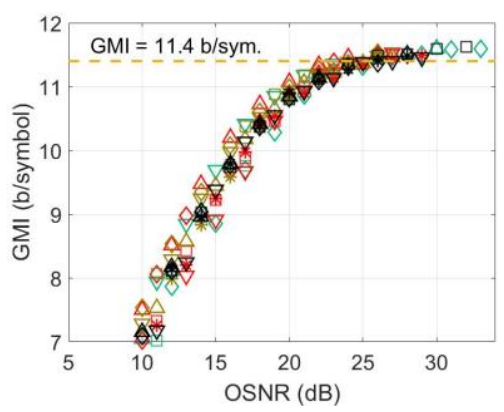

carriers for modulation. As seen in Fig. 5 (a), the quality factor, $\mathrm{Q}^{2}$, increases linearly and eventually saturate around $\mathrm{Q}^{2}=\sim 20$ $\mathrm{dB}$ at OSNR $>25 \mathrm{~dB}$, suggesting that OSNRs above $25 \mathrm{~dB}$ are sufficient for optical carriers in our system. To benchmark the performance of comb lines without distillation, we measure the required OSNR at $\mathrm{Q}^{2}=19.3 \mathrm{~dB}$, corresponding to $0.6 \mathrm{~b} /$ symbol or 5\% reduction of GMI for dual polarization 64QAM. At this indicative limit of $\mathrm{Q}^{2}=19.3 \mathrm{~dB}$ as illustrated with a yellow dashed line, we find that the required OSNR for all tested lines is in a range of 22-25 dB. When investigating the effect on BER (Fig. 5 (b)), BER mirrors the trend in $\mathrm{Q}^{2}$, dropping and saturating at BER below $10^{-2}$ when OSNR > $25 \mathrm{~dB}$. The threshold of $\mathrm{Q}^{2}=19.3 \mathrm{~dB}$ in the $\mathrm{Q}^{2}$ plot can translate into BER $=1.3 \times 10^{-2}$, and we see that the required OSNR is similar to that shown in the $\mathrm{Q}^{2}$ plot. This indicates that the noise can still be considered a Gaussian distributed noise field contribution after distillation. For the GMI plot in Fig. 5 (c), GMI shares a similar trend to $\mathrm{Q}^{2}$ and levels off at $\sim 11.6 \mathrm{~b} / \mathrm{symbol}$. With the reference for $\mathrm{Q}^{2}$ and BER in Fig. 5 (a), (b), this relates to the GMI limit of $11.4 \mathrm{~b} / \mathrm{symbol}$ regarded as a $5 \%$ reduction of the ideal achievable information rates of 64QAM at $12 \mathrm{~b} /$ symbol. Again, the required OSNR to achieve this limit also coincides with the ones belonging to $\mathrm{Q}^{2}$ and BER.

From the results presented in Fig. 5, we notice that if the OSNR of the tested lines is above $25 \mathrm{~dB}$, the performance can exceed our benchmark of a 5\% reduction in GMI. Therefore, we could regard OSNR $=25 \mathrm{~dB}$ as the lowest OSNR for our electro optic comb to achieve the benchmark without narrowband filtering for lines, which can be used to inform us of power requirements for optical frequency combs when used as carriers in optical communication systems.

(b)
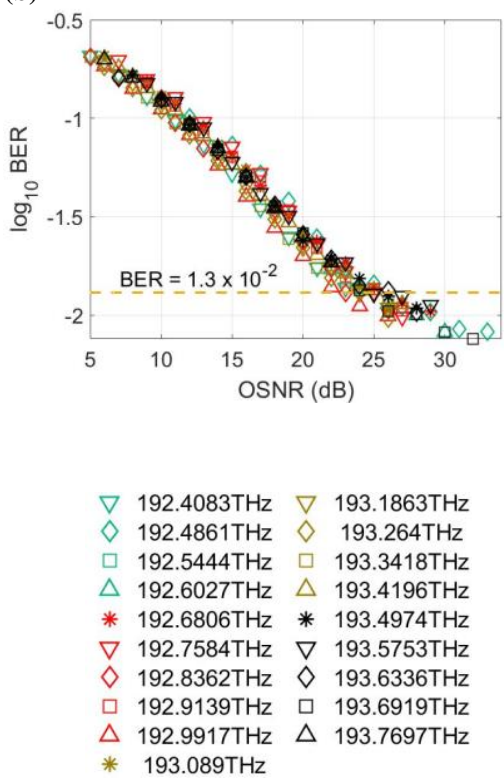

Fig. 5 Overlay performance of all bypassed tested carrier lines in terms of (a) Signal quality factor, $Q^{2}$, (b) BER, and (c) GMI, with dashed lines indicating limits of the $5 \%$ reduction of information rates in terms of the particular metrics 
(a)

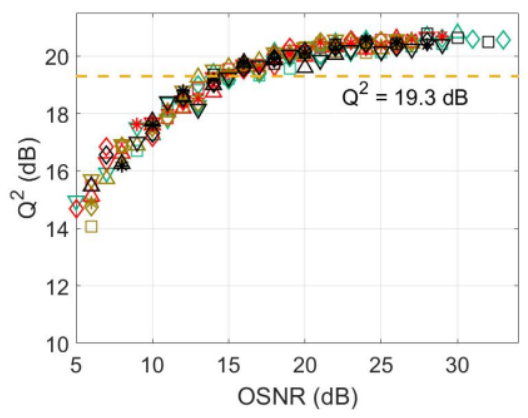

(c)

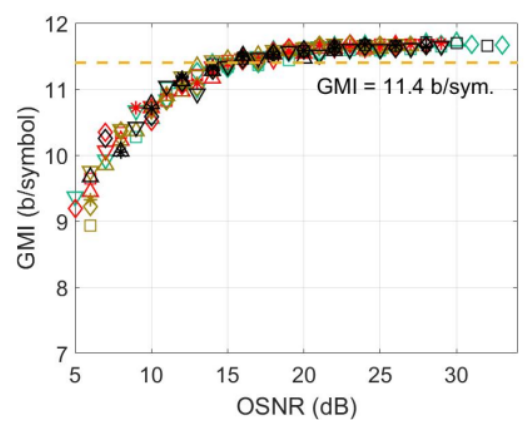

(b)

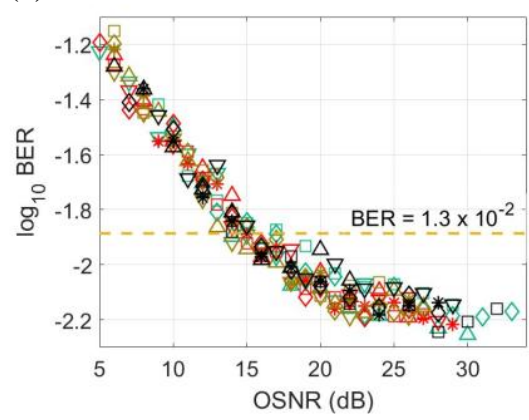

Fig. 6 Overlay performance of all tested carrier lines with distillation by the microring resonator in terms of (a) Signal quality factor, $\mathrm{Q}^{2}$, (b) BER, and (c) GMI, with dashed lines indicating limits of the $5 \%$ reduction of information rates in terms of the particular metrics

\section{B. With distillation}

Once passing the frequency comb through the microring resonator, that the performance of the lines improves compared to the undistilled case, as shown in Fig. 6 (a)-(c). With a closer look in Fig. 6 (a)-(b), we see that all traces saturate at $\mathrm{Q}^{2}$ almost $21 \mathrm{~dB}$ and BER below $9 \times 10^{-3}$ when OSNR $>20 \mathrm{~dB}$. Moreover, all lines require just $\sim 13-15 \mathrm{~dB}$ of OSNR to reach the limits. For GMI plot in Fig. 6 (c), it is seen that GMI also penetrates the limit of $11.4 \mathrm{~b} /$ symbol at OSNR $\sim 13-15 \mathrm{~dB}$ and begins flattening at GMI of 11.6 once OSNR is adjusted to be $>17 \mathrm{~dB}$.

From the plots of these metrics, we witness the improvement in performance when distilling comb lines with the resonator. This improvement is significant and can be up to $7 \mathrm{~dB}$ of $\mathrm{Q}^{2}$ when comparing Fig. 5 and 6 for OSNR below 25 $\mathrm{dB}$, i.e., where optical noise is dominant. However, once OSNR increases, the improvement is less noticeable since the optical noise plunges into the same level as transceiver noise which is the upper limit of the system. Moreover, ones can also state that comb distillation can extend the transceiver noise dominated performance $\sim 10 \mathrm{~dB}$ of OSNR as indicated in the difference of required OSNR at the 5\% information reduction limits for both cases, (i.e. required OSNR $\sim 22-25 \mathrm{~dB}$ for the bypassed case whereas $13-15 \mathrm{~dB}$ for the distilled case).

\section{Comparing performance with and without distillation}

To gain further insight for OSNR at $\mathrm{Q}^{2}=19.3 \mathrm{~dB}$, we interpolate $\mathrm{Q}^{2}$ values from each comb line for both cases and solve the fitting equations for required OSNR. Fig. 7 shows that required OSNR is generally flat for all tested frequencies with small fluctuations. The average required OSNR at the limits for carrier without and with distilled carriers are $\sim 24 \mathrm{~dB}$ and $\sim 15$ $\mathrm{dB}$, respectively, both with $\sim 1 \mathrm{~dB}$ variation. This implies a reduction of the required per line power by $9 \mathrm{~dB}$, suggesting that one could either introduce more lines for this comb source to support larger bandwidth of superchannel transmission, or reduce power of the seeding laser for the comb generation to minimize power consumption.

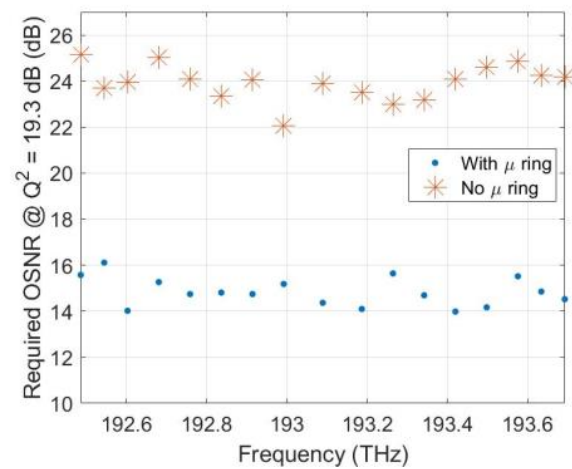

Fig. 7 Required OSNR at $\mathrm{Q}^{2}=19.3 \mathrm{~dB}$, for both bypassed and distilled carriers against frequency of comb lines.

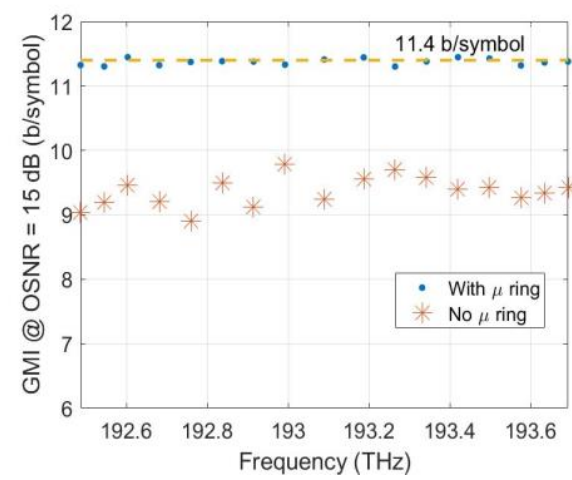

Fig. 8 GMI plot for distilled and bypassed carrier lines at OSNR of $15 \mathrm{~dB}$ with the limit of GMI $=11.4 \mathrm{~b} /$ symbol as indicated with the dashed line. 
Using the average required OSNR of $15 \mathrm{~dB}$ for the distilled carriers as a benchmark, we fit the curve and interpolate GMI values at this OSNR for the two cases, to estimate the increase in achievable bit rate through distillation. Fig. 8 shows that the values for the narrowband-filtered lines lie around the corresponding GMI limit of $11.4 \mathrm{~b} / \mathrm{symbol}$ with a small fluctuation of $\leq 0.1 \mathrm{~b} / \mathrm{symbol}$, while for the un-distilled lines GMI values lie around GMI $=9.5 \mathrm{~b} /$ symbol with a larger variation of $\sim 0.5 \mathrm{~b} / \mathrm{symbol}$. This indicates that distillation can improve GMI by about 2 bits/symbol for the 64QAM signals we investigate, showing that comb distillation can provide a real increase in achievable information rates for combs amplified from a low power seed.

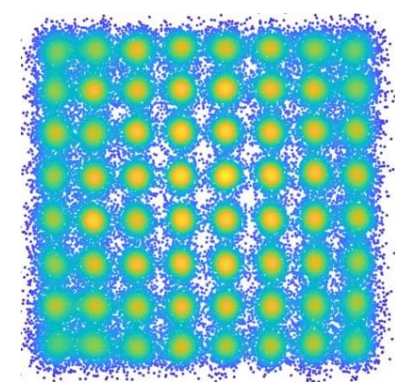

OSNR $=15 \mathrm{~dB}$ with $\mu \mathrm{rr}$

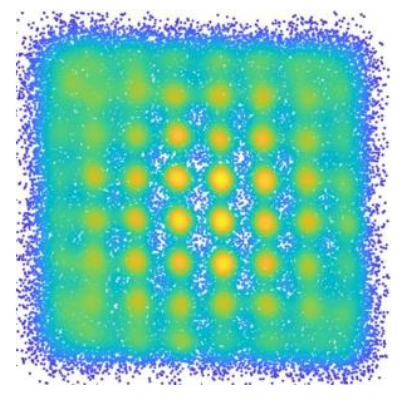

OSNR $=15 \mathrm{~dB}$ without $\mu \mathrm{rr}$
Fig. 9 Signal constellations as a results of distilled and bypassed carrier lines at $\mathrm{OSNR}=15 \mathrm{~dB}$.

In order to qualitatively visualize the impacts of comb distillation, Fig. 9 illustrates signal constellations distilled and undistilled carriers at OSNR of $15 \mathrm{~dB}$. Here, it can be seen that the signal constellation for the distilled carriers exhibits clear points with small distribution due to Gaussian noise in the

(a)

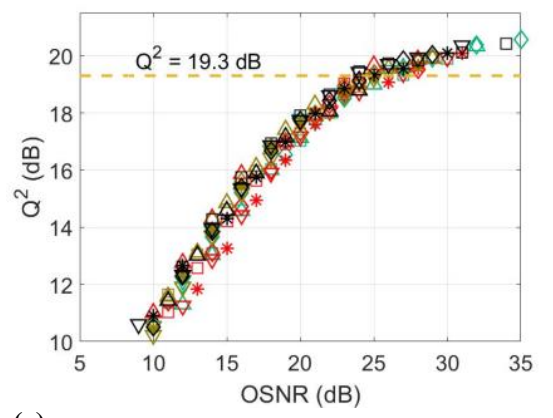

(c)

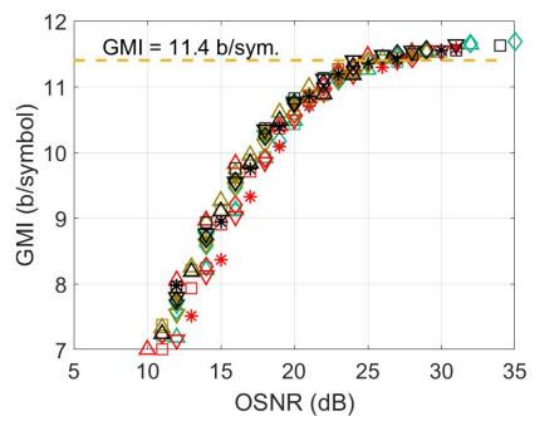

central part, with a noticeable increase invariance for the outer points. This contrast of variance with constellation point amplitude is more severe than the outer points in the case of undistilled lines. This can be explained by the fact that all symbol points have the same level of OSNR, i.e., amplitude to noise ratio, hence the further out from the origin the symbols are, the higher is noise.

\section{DEMONSTRATION OF COMB DISTILLATION FOR LOCAL OSCILLATORS}

In this section, we study possible impact comb distillation when applying the same approach as the previous section, but at the receiver side - i.e. to perform comb distillation for local oscillator (LO) lines.

\section{A. Without distillation}

After bypassed comb lines are fed into the LO port of the coherent receiver while ECL lines are used for carriers, the traces of performance metric reveal similar features to the case of bypassed carriers where all traces exhibit trends resulted from conventional noise loading. As seen in Fig. 10 (a)-(c), the traces saturate at the upper limits of $\mathrm{Q}^{2} \sim 20 \mathrm{~dB}, \mathrm{BER}<10^{-2}$, and GMI $\sim 11.6 \mathrm{~b} /$ symbol when OSNR reaches $\sim 27 \mathrm{~dB}$. When considering the $5 \%$ reduction of information rate limits, $\sim 22-25$ $\mathrm{dB}$ of OSNR for LO lines is required to achieve this limit. These values of OSNR coincide with the benchmarked results from bypassed carriers in Fig. 5. As a result, we could conclude that deployment of noisy comb lines at both transmission and reception sides after amplification sets the lower limit for perline or total comb power.

\section{B. With distillation}

To pursue the benefits of the microring resonator for LO line narrowband filtering, we purify the tested comb lines before

(b)
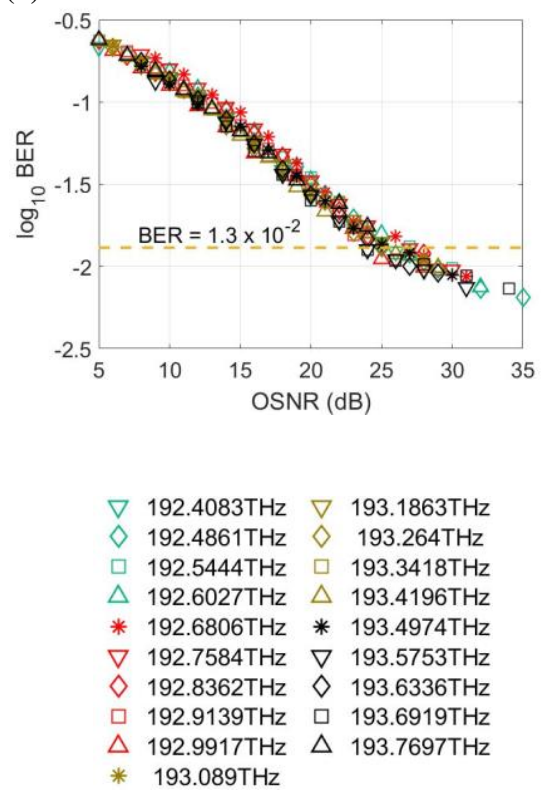

Fig. 10 Overlay performance of all tested LO lines without distillation in terms of (a) Signal quality factor, Q2 , (b) BER, and (c) GMI, with dashed lines indicating limits of the $5 \%$ reduction of information rates in terms of the particular metrics. 
(a)

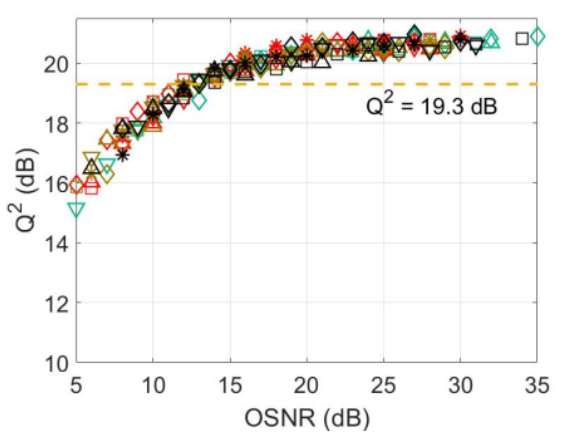

(c)

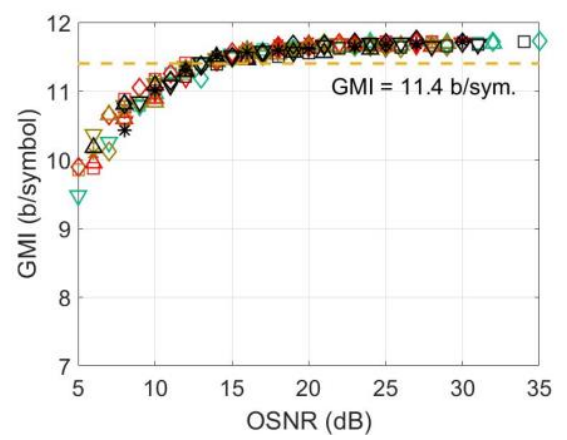

(b)

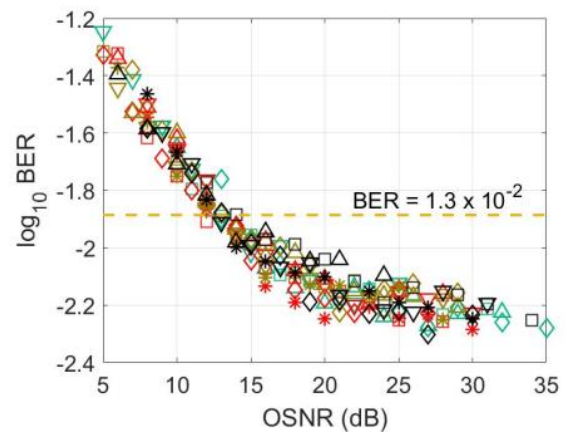

Fig. 11 Overlay performance of all tested LO lines with distillation by the microring resonator in terms of (a) Signal quality factor, Q², (b) BER, and (c) GMI, with dashed lines indicating limits of the $5 \%$ reduction of information rates in terms of the particular metrics.

using them as LOs. Fig. 11 (a)-(c) show the performance of the system after distilling the LO lines. Here, it is seen that all metrics become flat at $\mathrm{Q}^{2} \sim 20.5 \mathrm{~dB}$, BER $\sim 6 \times 10^{-3}$ when OSNR $=20 \mathrm{~dB}$, and for GMI, at $11.6 \mathrm{~b} / \mathrm{symbol}$ after OSNR reaches $17 \mathrm{~dB}$. Moreover, after comparing Fig. 11 (a) and Fig. 10 (a), we notice LO distillation can contribute to improvement in $\mathrm{Q}^{2}$ up to $\sim 8 \mathrm{~dB}$ especially in the optical noise dominated region (OSNR $\sim 10 \mathrm{~dB}$ ). When looking at the $5 \%$ reduction of information rates, the system requires only $12-14 \mathrm{~dB}$ of OSNR to attain this limit. This reduces the required OSNR for the comb by $\sim 12 \mathrm{~dB}$ compared with the bypassed case. Hence, we could suggest that LO distillation could help the system achieve the transceiver noise limit faster similar to the carrier distillation.

\section{Comparing performance with and without distillation}

We then plot the required OSNR at $\mathrm{Q}^{2}=19.3 \mathrm{~dB}$ for each tested frequency after interpolating each trace as shown in Fig. 12. We see that bypassed LO lines possess an overall flat profile of required OSNR with average required OSNR at $\sim 25 \mathrm{~dB}$, conforming to the one from Fig. 11 (a), with a variation of $1 \mathrm{~dB}$ while the distilled LO lines require just $13 \mathrm{~dB}$ with the same variation. This translates into $12 \mathrm{~dB}$ of OSNR reduction, suggesting that distillation could save per LO line power up to 15 times.

If we compare results of required OSNR for both carrier and LO distillation cases in Fig. 7 and 12, we could also state that narrowband filtering with the resonator tends to be more effective for comb lines deployed as LOs than carriers as the OSNR benefits caused by the resonator can be up to $12 \mathrm{~dB}$ for the LOs, while $9 \mathrm{~dB}$ for the carriers. Therefore, with these results, we conclude that it is more important to emphasis on mitigation for noise of LOs.

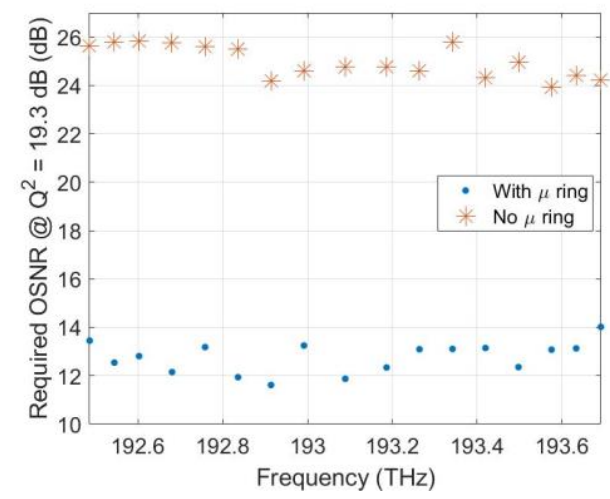

Fig. 12 Required OSNR at $\mathrm{Q}^{2}=19.3 \mathrm{~dB}$, for both bypassed and distilled LOs against frequency of comb lines.

To compare information rate of both bypassed and purified LOs at the benchmarked OSNR $=13 \mathrm{~dB}$, we plot the interpolated GMI at this OSNR of each line for both cases in Fig. 13. Again, we find similar patterns to the previous experiment where GMI for the distilled LOs fluctuates around the $11.4 \mathrm{~b} / \mathrm{symbol}$ limit with negligible deviation whereas GMI for the bypassed LOs is located well below $\sim 8 \mathrm{~b} / \mathrm{symbol}$. This indicates that the gain in achievable information rates is higher for a distilled LO, compared to the gain observed with a distilled carrier. We infer from this that the noise transfer through the LO is greater, possibly due to the constant high power of the local oscillator. We expect noise transfer via signal/LO beating in the coherent receiver. 


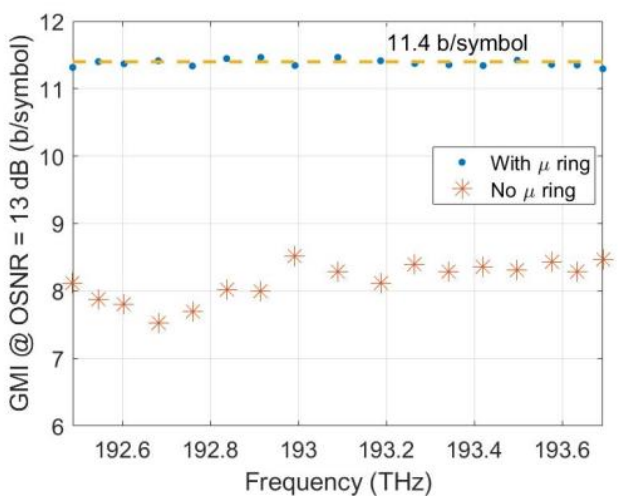

Fig. 13 GMI plot for distilled and bypassed LO lines at OSNR of $13 \mathrm{~dB}$ with the limit of GMI $=11.4 \mathrm{~b} / \mathrm{symbol}$ as indicated with the dashed line.
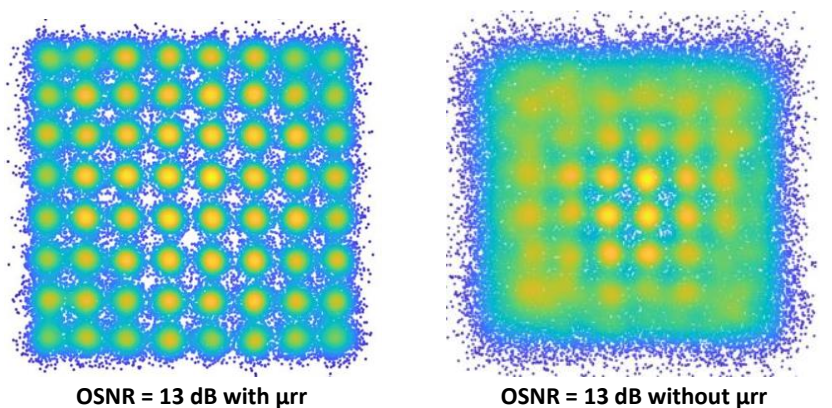

Fig. 14 Signal constellations as a results of distilled and bypassed LO lines at OSNR $=13 \mathrm{~dB}$.

Again, we can look qualitatively at the effects of LO distillation on signal constellations. Here in Fig. 14, the constellation at OSNR $=13 \mathrm{~dB}$ after passing LO lines through the resonator appears to be the ideal constellation with Gaussian distribution around the expected points due to noise. Nevertheless, the constellation due to the bypassed LO seems to be noisy especially at the edges of the constellation. This resembles results from the cases of carrier lines in Fig. 9 with an exception for required OSNR which is $2 \mathrm{~dB}$ lower, suggesting comb distillation plays a more important role for the LOs than for the carriers.

\section{Discussions}

As we have observed, comb distillation by the microring resonator offers a reduction in required OSNR at the threshold of $5 \%$ reduction for information rate by $\sim 9$ and $\sim 12 \mathrm{~dB}$ for carriers and LOs, respectively, with 64QAM scheme. These results might be lower for low-level modulation such as QPSK or 16QAM. This is because Euclidean distances between each symbol for those schemes are larger, causing the modulation formats to be more tolerant to errors than 64QAM, i.e., the schemes require lower OSNR to remain error-free $\left(\mathrm{BER} \leq 10^{-3}\right)$. Therefore, we expect the thresholds at which comb distillation becomes useful to change with modulation format.

While here we investigate comb distillation of carrier and LOs separately, we could propose line distillation with microring resonators for both carriers and LOs simultaneously. This may consist of two independent comb sources and microresonators, enabling realistic comb-based superchannel transmission. It must be noted that not only do resonance peaks, but also FSR of the resonators need to strictly match each other to avoid substantial frequency offset emerging at large-number modes relative to the central frequency as stated in [21]. This could still support high bandwidth transmission as highfrequency comb lines are still able to be utilized.

To further lower optical noise, higher $\mathrm{Q}$ microresonators $(\mathrm{Q}$ $\sim 10^{8}-10^{9}$ ), which are usually employed in nonlinear optics or comb generations [31]-[33] with resonance bandwidth in the order of $100 \mathrm{kHz}$ or sub megahertz, could be used to distill comb lines as their filter profiles can firmly fit the lines (linewidth $\sim 100 \mathrm{kHz}$ ), resulting in almost laser-like lines after distillation. However, this approach would be more sensitive to frequency mismatch between resonance peaks and comb lines as a result of dispersion in the resonators, shifting resonances from the equidistant grid, as illustrated in Fig. 15. Here, the comb lines match with the equidistant grid represented as the dashed lines. It is seen that the number of lines undergoing purification with narrow resonances is limited as the comb lines cannot reside in the shifted resonance lobes (due to dispersion) and are attenuated by the stopband part. However, with a tradeoff of in-line noise, distillation with wider resonances allows more comb lines to transmit through the drop port since the passband regions are wider, thus detuning of resonances due to chromatic dispersion has less effect. This may be a reason why we do not observe limitations in our comb bandwidth $(\sim 1.3$ $\mathrm{THz}$ ) after distillation.

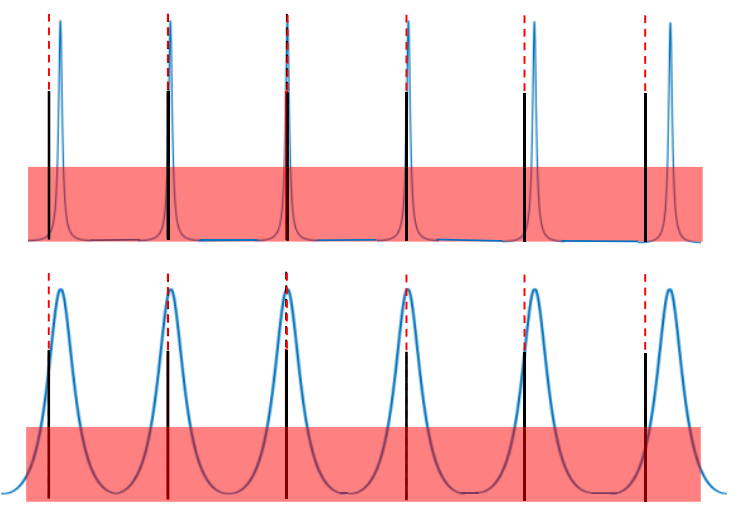

Fig. 15 Comb distillation by microring resonator with narrow bandwidth (top) and wide bandwidth (bottom) of resonances, where the comb lines are shown in black with noise floor in red and the equidistant grid is illustrated as the red dashed lines.

Although our proposed approach can lead to substantial noise reduction for comb lines, it still experiences limitations because line spacing is required to match with the fixed FSR of the resonator. This discourages flexibility of the source to be used for transmission of the arbitrary symbol rate. As a result, one can explore other potential approaches offering flexibility in line spacing adjustment. Two potential comb distillation options that are adaptable to comb spacing are distillation via stimulated Brillouin scattering (SBS), or through optical injection locking (OIL).

SBS can be used for comb line purification as it requires just another replica of the initial comb to be shifted by Brillouin 
frequency shift so that the initial comb lines are amplified via SBS, enabling line filtering with a bandwidth of $~ 30 \mathrm{MHz}[23]$, [24]. When scaling to many parallel lines, this flexibility to line spacing comes at the cost of high power for the pump light, and requires careful consideration of the dispersion in the SBS shift. Scaling to high comb line counts may require multiple watts of optical power [23], [24], which increases when compensating for dispersion in the SBS shift [34].

Another approach for comb purification is optical injection locking (OIL), where each comb line is injected into an individual slave laser so that the output frequency follows the line frequency. The approach can also support demultiplexing and amplification simultaneously by selecting the locking range to be smaller than the line spacing so that only one line is amplified while others are discarded [22]. With this principle, it is reported that low per-tone power combs can be amplified and employed in optical communications systems with better performance than the 'demultiplexing and amplification' method [35]. Similarly, scaling this approach to many parallel lines may have limitations, in that a new laser is required for each line, and each slave lasers may also requires stabilization to compensate frequency drifts, e.g., OIL phase lock loop [36] or pilot tone assisted OIL phase locked loop [37].

Reflecting on these alternative techniques, if it is possible to precisely set the comb line spacing used in a comb based optical communication system, the high-Q filter distillation approach we investigate here may provide to be desirable in terms of energy efficiency and complexity. If only a small number of lines are required to be distilled, such that the energy consumption and relative complexity of SBS- or OIL-based approaches is minimal, then the comb spacing need not be precisely set and those approaches may be a preferred alternative.

\section{CONCLUSION}

We propose an approach to reduce broadband noise around frequency comb lines based on a high $\mathrm{Q}$ microring resonator. Here, we test our approach using an EO comb and find that if the comb line spacing matches with the FSR of the resonator, narrowband filtering by resonances can give rise to substantial optical noise reduction. This is evidenced by our results for the lines over comb bandwidth where the required OSNR of bypassed carriers is $\sim 24 \mathrm{~dB}$ while for the distilled ones is $\sim 15$ $\mathrm{dB}$ at the $5 \%$ reduction of information rate limit $(\mathrm{GMI}=11.4$ b/symbol). With the same threshold, bypassed LOs require OSNR of $\sim 25 \mathrm{~dB}$ in contrast to the distilled LOs requiring OSNR just $\sim 13 \mathrm{~dB}$. According to significant noise reduction and parallelism, this suggests that a high $\mathrm{Q}$ microring resonator could be attached with chip-scale microcombs in order to simultaneously filter in-line noise for comb lines before being deployed to support high bandwidth optical communications.

\section{REFERENCE}

[1] N. Picqué and T. W. Hänsch, "Frequency comb spectroscopy," Nat. Photonics, vol. 13, no. 3, pp. 146-157, 2019, doi: 10.1038/s41566-0180347-5.

[2] G. Millot et al., "Frequency-agile dual-comb spectroscopy," Nat. Photonics, vol. 10, no. 1, pp. 27-30, 2016, doi: 10.1038/nphoton.2015.250.
[3] M. Yu, Y. Okawachi, A. G. Griffith, N. Picqué, M. Lipson, and A. L. Gaeta, "Silicon-chip-based mid-infrared dual-comb spectroscopy," Nat. Commun., vol. 9, no. 1, p. 1869, 2018, doi: 10.1038/s41467-018-04350-1.

[4] J. Feldmann et al., "Parallel convolutional processing using an integrated photonic tensor core," Nature, vol. 589, no. 7840, pp. 52-58, 2021, doi: 10.1038/s41586-020-03070-1.

[5] X. Xu et al., "11 TOPS photonic convolutional accelerator for optical neural networks," Nature, vol. 589, no. 7840, pp. 44-51, 2021, doi: 10.1038/s41586-020-03063-0.

[6] M. Mazur, A. Lorences-Riesgo, J. Schröder, P. A. Andrekson, and M. Karlsson, "High Spectral Efficiency PM-128QAM Comb-Based Superchannel Transmission Enabled by a Single Shared Optical Pilot Tone," J. Light. Technol., vol. 36, no. 6, pp. 1318-1325, Mar. 2018, doi: 10.1109/JLT.2017.2786750.

[7] A. Pasquazi et al., "Micro-combs: A novel generation of optical sources," Phys. Rep., vol. 729, pp. 1-81, 2018.

[8] A. L. Gaeta, M. Lipson, and T. J. Kippenberg, "Photonic-chip-based frequency combs," Nat. Photonics, vol. 13, no. 3, pp. 158-169, 2019, doi: 10.1038/s41566-019-0358-x.

[9] T. J. Kippenberg, A. L. Gaeta, M. Lipson, and M. L. Gorodetsky, "Dissipative Kerr solitons in optical microresonators," Science (80-. )., vol. 361, no. 6402, 2018, doi: 10.1126/science.aan8083.

[10] L. Razzari et al., "CMOS-compatible integrated optical hyper-parametric oscillator," Nat. Photonics, vol. 4, no. 1, p. 41, 2010.

[11] X. Xu, M. Tan, J. Wu, R. Morandotti, A. Mitchell, and D. J. Moss, "Microcomb-Based Photonic RF Signal Processing," IEEE Photonics Technol. Lett., vol. 31, no. 23, pp. 1854-1857, Dec. 2019, doi: 10.1109/LPT.2019.2940497.

[12] J. Wu et al., "RF Photonics: An Optical Microcombs' Perspective," IEEE J. Sel. Top. Quantum Electron., vol. 24, no. 4, pp. 1-20, Jul. 2018, doi: 10.1109/JSTQE.2018.2805814.

[13] P. Marin-Palomo et al., "Microresonator-based solitons for massively parallel coherent optical communications," Nature, vol. 546, no. 7657, pp. 274-279, 2017, doi: 10.1038/nature22387.

[14] J. Pfeifle et al., "Optimally Coherent Kerr Combs Generated with Crystalline Whispering Gallery Mode Resonators for Ultrahigh Capacity Fiber Communications," Phys. Rev. Lett., vol. 114, no. 9, p. 93902, Mar. 2015, doi: 10.1103/PhysRevLett.114.093902.

[15] B. Corcoran et al., "Ultra-dense optical data transmission over standard fibre with a single chip source," Nat. Commun., vol. 11, no. 1, p. 2568, 2020, doi: 10.1038/s41467-020-16265-x.

[16] T. Herr et al., "Universal formation dynamics and noise of Kerr-frequency combs in microresonators," Nat. Photonics, vol. 6, no. 7, pp. 480-487, 2012, doi: 10.1038/nphoton.2012.127.

[17] S. J. Herr et al., "Frequency comb up- and down-conversion in synchronously driven $\chi^{2}$ optical microresonators," Opt. Lett., vol. 43, no. 23, pp. 5745-5748, Dec. 2018, doi: 10.1364/OL.43.005745.

[18] M. Zhang et al., "Broadband electro-optic frequency comb generation in a lithium niobate microring resonator," Nature, vol. 568, no. 7752, pp. 373377, 2019, doi: 10.1038/s41586-019-1008-7.

[19] A. J. Metcalf, V. Torres-Company, D. E. Leaird, and A. M. Weiner, "HighPower Broadly Tunable Electrooptic Frequency Comb Generator," IEEE J. Sel. Top. Quantum Electron., vol. 19, no. 6, pp. 231-236, Nov. 2013, doi: 10.1109/JSTQE.2013.2268384.

[20] M. Kourogi, K. Nakagawa, and M. Ohtsu, "Wide-span optical frequency comb generator for accurate optical frequency difference measurement," IEEE J. Quantum Electron., vol. 29, no. 10, pp. 2693-2701, Oct. 1993, doi: $10.1109 / 3.250392$.

[21] V. Torres-Company et al., "Laser Frequency Combs for Coherent Optical Communications," J. Light. Technol., vol. 37, no. 7, pp. 1663-1670, Apr. 2019, [Online]. Available: http://jlt.osa.org/abstract.cfm?URI=jlt-37-71663.

[22] Z. Liu and R. Slavík, "Optical Injection Locking: From Principle to Applications," J. Light. Technol., vol. 38, no. 1, pp. 43-59, Jan. 2020, doi: 10.1109/JLT.2019.2945718.

[23] A. Choudhary et al., "On-chip Brillouin purification for frequency combbased coherent optical communications," Opt. Lett., vol. 42, no. 24, pp. 5074-5077, Dec. 2017, doi: 10.1364/OL.42.005074.

[24] M. Pelusi et al., "Low noise frequency comb carriers for 64-QAM via a Brillouin comb amplifier," Opt. Express, vol. 25, no. 15, pp. 17847-17863, Jul. 2017, doi: 10.1364/OE.25.017847.

[25] K. Beha, D. C. Cole, P. Del'Haye, A. Coillet, S. A. Diddams, and S. B. Papp, "Electronic synthesis of light," Optica, vol. 4, no. 4, pp. 406-411, Apr. 2017, doi: 10.1364/OPTICA.4.000406.

[26] B. Corcoran et al., "Overcoming low-power limitations on optical 
frequency combs using a micro-ring resonator," in Optical Fiber Communication Conference (OFC) 2020, 2020, p. T4G.5, doi: 10.1364/OFC.2020.T4G.5.

[27] W. Bogaerts et al., "Silicon microring resonators," Laser Photon. Rev., vol. 6, no. 1, pp. 47-73, Jan. 2012, doi: https://doi.org/10.1002/lpor.201100017.

[28] D. J. Moss, R. Morandotti, A. L. Gaeta, and M. Lipson, "New CMOScompatible platforms based on silicon nitride and Hydex for nonlinear optics," Nat. Photonics, vol. 7, no. 8, pp. 597-607, 2013, doi: 10.1038/nphoton.2013.183.

[29] M. Ferrera et al., "Low-power continuous-wave nonlinear optics in doped silica glass integrated waveguide structures," Nat. Photonics, vol. 2, no. 12, pp. 737-740, 2008, doi: 10.1038/nphoton.2008.228.

[30] K. Kikushima and H. Yoshinaga, "Distortion due to gain tilt of erbiumdoped fiber amplifiers," IEEE Photonics Technol. Lett., vol. 3, no. 10, pp. 945-947, Oct. 1991, doi: 10.1109/68.93272.

[31] T. Herr et al., "Temporal solitons in optical microresonators," Nat. Photonics, vol. 8, no. 2, pp. 145-152, 2014, doi: 10.1038/nphoton.2013.343.

[32] P. Del'Haye, S. A. Diddams, and S. B. Papp, "Laser-machined ultra-highQ microrod resonators for nonlinear optics," Appl. Phys. Lett., vol. 102, no. 22, p. 221119, 2013, doi: 10.1063/1.4809781.

[33] T. J. Kippenberg, S. M. Spillane, and K. J. Vahala, "Kerr-Nonlinearity Optical Parametric Oscillation in an Ultrahigh Q Toroid Microcavity," Phys. Rev. Lett., vol. 93, no. 8, p. 83904, Aug. 2004, doi: 10.1103/PhysRevLett.93.083904.

[34] A. Zarifi, M. Merklein, Y. Liu, A. Choudhary, B. J. Eggleton, and B. Corcoran, "EDFA-band Coverage Broadband SBS Filter for Optical Carrier Recovery," in 2020 Conference on Lasers and Electro-Optics Pacific Rim (CLEO-PR), Aug. 2020, pp. 1-2, doi: 10.1364/CLEOPR.2020.C9G_3.

[35] A. Albores-Mejia, T. Kaneko, E. Banno, K. Uesaka, H. Shoji, and H. Kuwatsuka, "Optical-Comb-Line Selection from a Low-Power/LowOSNR Comb using a Low-Coherence Semiconductor Laser for Flexible Ultra-Dense Short Range Transceivers," in Optical Fiber Communication Conference, 2015, p. W2A.23, doi: 10.1364/OFC.2015.W2A.23.

[36] A. C. Bordonalli, C. Walton, and A. J. Seeds, "High-Performance Phase Locking of Wide Linewidth Semiconductor Lasers by Combined Use of Optical Injection Locking and Optical Phase-Lock Loop," J. Light. Technol., vol. 17, no. 2, p. 328, Feb. 1999, [Online]. Available: http://jlt.osa.org/abstract.cfm?URI=jlt-17-2-328.

[37] Z. Liu, J. Kim, D. S. Wu, D. J. Richardson, and R. Slavík, "Homodyne OFDM with Optical Injection Locking for Carrier Recovery," J. Light. Technol., vol. 33, no. 1, pp. 34-41, Jan. 2015, doi: 10.1109/JLT.2014.2369994. 